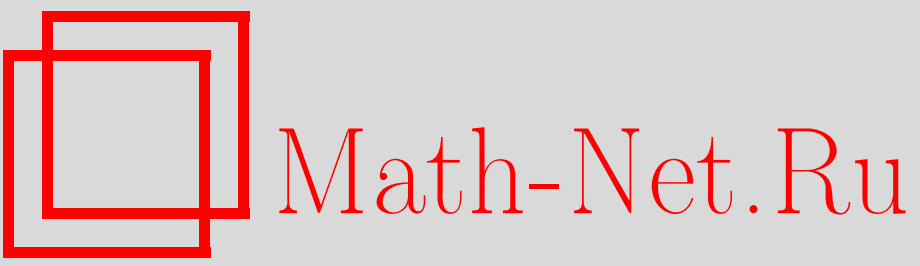

С. В. Савченко, О спектральных свойствах неразложимой неотрицательной матрицы и ее главных подматриц копорядка один, УМH, 2000, том 55, выпуск 1, 191-192

DOI: https://doi.org/10.4213/rm260

Использование Общероссийского математического портала Math-Net.Ru подразумевает, что вы прочитали и согласны с пользовательским соглашением

http://www.mathnet.ru/rus/agreement

Параметры загрузки:

IP: 34.229 .45 .116

26 апреля 2023 г., 17:53:56 


\title{
О СПЕКТРАЛЬНЫХ СВОЙСТВАХ НЕРАЗЛОЖИМОЙ НЕОТРИЦАТЕЛЬНОЙ МАТРИЦЫ И ЕЕ ГЛАВНЫХ ПОДМАТРИЦ КОПОРЯДКА ОДИН
}

\author{
C. В. Савчеhко
}

Пусть $\lambda$ - собственное значение подматрицы $A-v$, полученной из конечной матрицы $A$ с элементами $A\left(w, w^{\prime}\right)$ вычеркиванием $v$-столбца и $v$-строки. Упорядочим по убыванию порядки соответствующих ему жордановых клеток: $n_{1}=\cdots=n_{d}>n_{d+1} \geqslant \cdots \geqslant n_{k}$. Пусть $\widehat{\xi}_{p}$, $p=1, \ldots, d,-$ векторы, порождаюшие жордановы цепочки максимального порядка, и $\xi_{p}, p=$ $1, \ldots, d,-$ соответствуюшие им собственные векторы: $(A-v-\lambda E)^{n_{p}-1} \widehat{\xi_{p}}=\xi_{p}$, где $E$ - единичная матрица. Пусть $\eta_{p}, p=1, \ldots, d,-$ собственные векторы транспонированной к $A-v$ матрицы, принадлежащие жордановым цепочкам максимального порядка, отвечающим собственному значению $\lambda$. Определим однозначно по $\widehat{\xi}$ векторы $\eta_{p}$ (возможность такого выбора см. в п. 5 [1]) условием $\left(\widehat{\xi}_{p^{\prime}}, \eta_{p}\right)=\delta_{p^{\prime} p}$, где $\delta_{p^{\prime} p}-$ символ Кронекера и $(\xi, \eta)=\sum_{w} \xi(w) \eta(w)$. Обозначим через $\xi_{v}$ и $\eta_{v}$ векторы с координатами $\xi_{v}(w)=A(v, w)$ и $\eta_{v}(w)=A(w, v)$ (здесь $\left.w \neq v\right)$.

Теорема 1. Множество порядков жордановых клеток, соответствующих точке $\lambda$ как собственному значению матрицы $A$, совпадает с совокупностью чисел $\left\{n_{2}, \ldots, n_{k}\right\}$, если и только если

$$
\sum_{p=1}^{d}\left(\xi_{v}, \xi_{p}\right)\left(\eta_{v}, \eta_{p}\right) \neq 0
$$

Переход от $A-v$ к $A$, при котором у фиксированного собственного значения $\lambda$ "пропадает" одна максимальная жорданова клетка, а порядки остальных не меняются, по понятным причинам, мы будем называть типичным для $\lambda$. Можно показать, что типичный переход одновременно является и максимально возможным: больше чем на $n_{1}$ и 1 соответственно алгебраическая и геометрическая кратности собственного значения $\lambda$ уменьшиться не могут.

Обозначим через $\lambda(A)$ собственное значение неотрицательной матрицы $A$ (так в дальнейшем мы будем называть матрицу с неотрицательными элементами), совпадающее с ее спектральным радиусом (см. главу XIII [2]). Определим граф $G(A)$ матрицы $A$ по следующему правилу: из $w$ в $w^{\prime}$ выходит ребро тогда и только тогда, когда $A\left(w, w^{\prime}\right) \neq 0$. Если граф $G(A)$ является связным, то матрица $A$ назьвается неразложимой.

Теорема 2. Пусть $A$ - конечная неразложимая неотрицательная матрица. Тогда при любом $v$ переход от $A-v \kappa A$ является типичным для $\lambda=\lambda(A-v)$.

Индекс $v$ называется минимальным, если $\lambda(A-v)=\min _{w} \lambda(A-w)$.

Теорема 3. Пусть А - конечная неразложимая неотрицательная матрица. Тогда если индекс $v$ не является минимальным, то алгебрачческая кратность собственного значения $\lambda(A-v)$ матрицы $A-v$ равна единице. В случае минимального индекса $v$ число $k$ жордановых клеток, соответствующих собственному значению $\lambda(A-v)$ матрицы $A-v$, также не зависит от конкретного выбора $v$. Для любых двух минимальных индексов упорядоченные по убъванию порядки $n_{i}$ жордановых клеток совпадают при $i=2, \ldots, k$. Что касается порядков $n_{1}$ максимальных жсордановых клеток, то они могут отличаться друг от друга не более чем на единичу.

В силу теорем 2 и 3 только для минимального индекса $v$ число $\lambda(A-v)$ может быть собственным значением матрицы $A$ (то, что во всем интервале $(\lambda(A-v), \lambda(A))$ нет точек спектра матрицы $A$ с положительными элементами, было известно еще робениусу; общий случай см. в [3]). Теорема 2 позволяет сформулировать следующий критерий: точка $\lambda(A-v)$ принадлежит спектру неразложимой неотрицательной матрицы $A$, если и только если в графе $G(A-v)$ отсутствует путь, проходящий через все его подграфы $G\left((A-v)_{i}\right)$, где $(A-v)_{i}$ - неразложимые подматрицы 
матрицы $A-v$, для которых $\lambda\left((A-v)_{i}\right)=\lambda(A-v)$ (по теореме 6.3 [4] существование такого пути равносильно наличию всего одной жордановой клетки).

Для бесконечной неотрицательной матрицы $A$ число $\lambda(A)$ определим как супремум спектральных радиусов ее конечных подматриц. В дальнейшем мы будем рассматривать только устойчиво положительно-рекуррентные матрицы, т.е. неразложимые неотрицательные матрицы $A, \mathrm{y}$ которых диагональньй элемент матричнозначной функции $(E-z A)^{-1}$ имеет полюс в точке $z=\lambda(A)^{-1}$ (см. [5]). В условии следующей теоремы предполагается, что матрица $A-v$ получена из $A$ заменой нулями элементов $v$-столбца и $v$-строки.

Теорема 4. Для любой устойчиво положительно-рекуррентной матрицъ $А$ найдется такая положительная мера $\mu$ на множсестве ее индексов, что при любом $v$, за исключением, быть может, әлементов некоторого конечного множества, действие

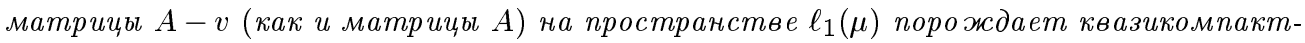
ный оператор. Его спектральный радиус совпадает с $\lambda(A-v)$, и в этой точке он имеет простое собственное значение, т.е. изолированное собственное значение алгебраической кратности единица (это утверждение с заменой $\lambda(A-v)$ на $\lambda(A)$ верно и для $A$, причем интервал $[\lambda(A-v), \lambda(A))$ не принадлежит спектру $A)$.

Если неотрицательная матрица $A$ симметрична и $\lambda(A)<\infty$, то она определяет на пространстве $\ell_{2}$ ограниченный самосопряженньй оператор, максимальная точка спектра которого совпадает с $\lambda(A)$. В этом случае условие устойчивой положительной рекуррентности эквивалентно изолированности точки $\lambda(A)$ от остальной части спектра (ее кратность как собственного значения равна единице и координаты соответствующего собственного вектора положительны). Для произвольной эрмитовой матрицы $A$, порождающей ограниченный самосопряженный оператор (его мы также обозначим через $A$ ), число $\lambda(A)$ определим как максимальную точку спектра этого оператора. Очевидно, понятие минимального индекса здесь также имеет смысл. При помощи принципа минимакса (см. [6]) легко показать, что если $\lambda(A)$ - простое собственное значение и все координаты собственного вектора отличны от нуля, то множество минимальных индексов матрицы $A$ конечно, и для любого ее неминимального индекса $v$ точка $\lambda(A-v)$ не принадлежит спектру оператора $A$ и, следовательно, является простым собственным значением $A-v$.

Теорема 5. Пусть А- симметрическая устойчиво положительно-рекуррентная матрица и $v-е е$ минимальный индекс. Тогда кратность $k$ собственного значения $\lambda(A-v)$ при переходе от $A-v \kappa A$ уменьшается на единицу (если $k=0$, то $\lambda(A-v)-$ точка непрерывного спектра обоих операторов). В случае, когда $k \geqslant 2$, минимальный индекс единствен.

\section{СПИСОК ЛИТЕРАТУРЫ}

[1] Келдыш М. В. // УМН. 1971. Т. 26. №4. С. 15-41. [2] Гантмахер Ф. Р. Теория матриц. М.: Наука, 1967. [3] Friedland S., Nabben R. // Linear Algebra Appl. 1997. V. 255. P. 303-313. [4] Schneider H. // Linear Algebra Appl. 1986. V. 84. P. 161-189. [5] Гуревич Б. М., Савченко С. В. // УМН. 1998. Т. 53. № 2. С. 3-106. [6] Рид М., Саймон Б. Методы современной математической физики. T. IV. М.: Мир, 1982. 